\title{
Nearly Gorenstein rings arising from finite graphs
}

\author{
Takayuki Hibi * \\ Department of Pure and Applied Mathematics \\ Graduate School of Information Science and Technology \\ Osaka University \\ Suita, Osaka 565-0871, Japan \\ hibi@math.sci.osaka-u.ac.jp \\ Dumitru I. Stamate \\ Faculty of Mathematics and Computer Science \\ University of Bucharest \\ Str. Academiei 14 \\ Bucharest - 010014, Romania \\ dumitru.stamate@fmi.unibuc.ro
}

Submitted: Apr 8, 2021; Accepted: Jul 2, 2021; Published: July 30, 2021

(C) The authors. Released under the CC BY-ND license (International 4.0).

\begin{abstract}
The classification of complete multipartite graphs whose edge rings are nearly Gorenstein as well as that of finite perfect graphs whose stable set rings are nearly Gorenstein is achieved.

Mathematics Subject Classifications: 13H10, 05E40, 05C17, 05C69, 14M25, 06A11
\end{abstract}

Gorenstein graded algebras associated to combinatorial objects like graphs or simplicial complexes have attracted a lot of interest. See, e.g., [5], [16], [2]. Recently several extensions of the class of Gorenstein rings (inside the class of Cohen-Macaulay rings) have been discussed in, e.g., [6], [7], hence it is natural to search for the combinatorial counterpart.

According to [7], when $R$ is a Cohen-Macaulay graded $\mathbb{K}$-algebra over the field $\mathbb{K}$ with canonical module $\omega_{R}$, it is called nearly Gorenstein if the canonical trace ideal $\operatorname{tr}\left(\omega_{R}\right)$ contains the maximal graded ideal $\mathfrak{m}_{R}$ of $R$. Here $\operatorname{tr}\left(\omega_{R}\right)$ is the ideal generated by the image of $\omega_{R}$ through all homomorphism of $R$-modules into $R$. As $\operatorname{tr}\left(\omega_{R}\right)$ describes the

\footnotetext{
*Partially supported by JSPS KAKENHI 19H00637.
} 
non-Gorenstein locus of $R$ ([7, Lemma 2.1]), one has $\operatorname{tr}\left(\omega_{R}\right)=R$ if and only if $R$ is a Gorenstein ring.

In the present paper we initiate the study of nearly Gorenstein rings belonging to two classes of algebras associated to graphs. Throughout, $\mathbb{K}$ is any field. Assume $G$ is a simple graph (it possesses no loops or multiple edges) with vertex set $V(G)=[d]:=\{1, \ldots, d\}$.

The edge ring $\mathbb{K}[G]$ is the $\mathbb{K}$-subalgebra of the polynomial ring $\mathbb{K}\left[x_{1}, \ldots, x_{d}\right]$ generated by the monomials $x_{i} x_{j}$ for all edges $\{i, j\} \in E(G)$. When $V(G)$ can be partitioned $V(G)=\sqcup_{k=1}^{n} V_{k}$ with $n \geqslant 2$ and $\left|V_{k}\right|=r_{k}$ for $k=1, \ldots, n$ such that $E(G)$ consists of all the pairs $\{i, j\}$ with $i \in V_{a}$ and $j \in V_{b}$ for $1 \leqslant a<b \leqslant n$, we say that $G$ is a complete multipartite graph of type $r_{1}, \ldots, r_{n}$ which is denoted $K_{r_{1}, \ldots, r_{n}}$. Related algebraic properties for these graphs have been recently studied in [10] and [11]. In Proposition 5 and in Theorem 6 we prove the following result.

Theorem A. Assume $G=K_{r_{1}, \ldots, r_{n}}$. Set $R=\mathbb{K}[G]$. Then

1. if $n=2$ and $1 \leqslant r_{1} \leqslant r_{2}$, the ring $R$ is nearly Gorenstein if and only if $r_{1}=1$, or $r_{2} \in\left\{r_{1}, r_{1}+1\right\}$.

2. if $n \geqslant 3$ the ring $R$ is nearly Gorenstein if and only if $R$ is Gorenstein.

Since Ohsugi and Hibi in [14] have explicitly listed the complete multipartite graphs whose edge ring is Gorenstein (see Theorem 1 below), Theorem A offers a full description for the nearly Gorenstein property, as well.

The other class of algebras we consider deals with the stable sets in $G$. A nonempty set $W$ of vertices is called stable (or independent) if there is no edge $\{i, j\}$ in $G$ with $i, j \in W$. The stable set ring of $G$ denoted $\operatorname{Stab}_{\mathbb{K}}(G)$ is the $\mathbb{K}$-subalgebra in the polynomial ring $\mathbb{K}\left[x_{1}, \ldots, x_{d}, t\right]$ generated by those monomials $\left(\prod_{i \in W} x_{i}\right) \cdot t$ with $W$ any stable set in $G$. When $G$ is a perfect graph, it is known [15] that $\operatorname{Stab}_{\mathbb{K}}(G)$ is Cohen-Macaulay, and that it is Gorenstein if and only if all maximal cliques of $G$ have the same cardinality [16]. Recall that a set $C \subset V(G)$ is called a clique if the subgraph induced by $C$ is a complete graph.

The size of the maximal cliques in $G$ is also relevant to describe in Theorem 13 for which perfect graphs the algebra $\operatorname{Stab}_{\mathbb{K}}(G)$ is nearly Gorenstein. We prove the following.

Theorem B. Let $G$ be a perfect graph and $G_{1}, \ldots, G_{s}$ its connected components. Let $\delta_{i}$ denote the maximal cardinality of cliques of $G_{i}$. Then $\operatorname{Stab}_{\mathbb{K}}(G)$ is nearly Gorenstein if and only if for each $G_{i}$ its maximal cliques have the same cardinality and $\left|\delta_{i}-\delta_{j}\right| \leqslant 1$ for $1 \leqslant i<j \leqslant s$.

To prove Theorems $\mathrm{A}$ and $\mathrm{B}$ we observe that the algebras $R$ which occur are CohenMacaulay domains, so $\omega_{R}$ can be identified with an ideal in $R$. By [7, Lemma 1.1], its trace can be computed as

$$
\begin{aligned}
\operatorname{tr}\left(\omega_{R}\right) & =\omega_{R} \cdot \omega_{R}^{-1}, \text { where } \\
\omega_{R}^{-1} & =\left\{x \in Q(R): x \cdot \omega_{R} \subseteq R\right\}
\end{aligned}
$$

is the anti-canonical ideal of $R$ and $Q(R)$ denotes the field of fractions of $R$.

We refer the reader to [1] and [2] for the undefined graph or algebraic notions. 


\section{Edge rings}

In this section unless stated otherwise $G=K_{r_{1}, \ldots r_{n}}$ is the complete multipartite graph on $[d]$ with vertices partitioned $V(G)=V_{1} \sqcup \cdots \sqcup V_{n}, n \geqslant 2,\left|V_{k}\right|=r_{k}$ for all $k$. In this context $d=\sum_{k=1}^{n} r_{k}$ and without loss of generality, we will always assume that $1 \leqslant r_{1} \leqslant \ldots \leqslant r_{n}$.

The graph $G$ satisfies the so called odd cycle condition, i.e. for any two odd cycles in $G$ which have no common vertex there is a bridge between them. Indeed, when $n=2$ there is no odd cycle and anything to prove. Assume $n \geqslant 3$, and $C_{1}$ and $C_{2}$ be two disjoint odd cycles in $G$. Since $G$ is multipartite, each of these contains vertices from at least two of the components $V_{1}, \ldots, V_{n}$, so one finds $v \in C_{1} \cap V_{a}$ and $w \in C_{2} \cap V_{b}$ with $a \neq b$. Then $v w$ is an edge in $G$ and a bridge between $C_{1}$ and $C_{2}$. Consequently, by [13] the edge ring

$$
R=\mathbb{K}[G]=\mathbb{K}\left[x_{i} x_{j}: i \in V_{a}, j \in V_{b}, 1 \leqslant a<b \leqslant n\right] \subset \mathbb{K}\left[x_{1}, \ldots, x_{d}\right]
$$

is normal, hence a Cohen-Macaulay domain ([12]). Before we address the nearly Gorenstein property, we recall that Ohsugi and Hibi [14] classified the complete multipartite edge rings which are Gorenstein. With notation as above, their result is the following.

Theorem 1. (Ohsugi, Hibi [14, Remark 2.8]) The edge ring of the complete multipartite graph $K_{r_{1}, \ldots, r_{n}}$ is Gorenstein if and only if

1. $n=2$ and $\left(r_{1}, r_{2}\right) \in\{(1, m),(m, m): m \geqslant 1\}$, or

2. $n=3$ and $1 \leqslant r_{1} \leqslant r_{2} \leqslant r_{2} \leqslant 2$, or

3. $n=4$ and $r_{1}=r_{2}=r_{3}=r_{4}=1$.

For some complete multipartite graphs the edge ring fits into classes of algebras for which the nearly Gorenstein property is already understood.

Example 2. When $r_{1}=\cdots=r_{n}=1$, the edge ring $R$ is the squarefree Veronese subalgebra of degree 2 in the polynomial ring $\mathbb{K}\left[x_{1}, \ldots, x_{n}\right]$, and according to $[7$, Theorem 4.14], $R$ is nearly Gorenstein if and only if it is Gorenstein. The latter property holds if and only if $n \leqslant 4$, by using work of De Negri and Hibi [5], or Bruns, Vasconcelos and Villarreal [3].

Example 3. According to Higashitani and Matsushita [10, Proposition 2.2], when $n=2$, or when $n=3$ and $r_{1}=1$, the corresponding edge ring is isomorphic to a Hibi ring, and for the latter the nearly Gorenstein property is described in [7]. We refer to [9] for background on Hibi rings.

Theorem 4 ([7, Theorem 5.4], [9]). Let $P$ be a finite poset. Then the Hibi ring $R$ of the distributive lattice of the order ideals in $P$ is nearly Gorenstein if and only if $P$ is the disjoint union of pure connected posets $P_{1}, \ldots, P_{q}$ such that $\left|\operatorname{rank}\left(P_{i}\right)-\operatorname{rank}\left(P_{j}\right)\right| \leqslant 1$ for $1 \leqslant i<j \leqslant q$.

In particular, $R$ is a Gorenstein ring if and only if $P$ is pure. 
Based on that, when $G$ is a complete bipartite graph we obtain the following classification.

Proposition 5. Let $G=K_{r_{1}, r_{2}}$ be the complete bipartite graph with $1 \leqslant r_{1} \leqslant r_{2}$. Then the edge ring $\mathbb{K}[G]$ is nearly Gorenstein if and only if $r_{1}=1$, or $r_{1} \geqslant 2$ and $r_{2} \in\left\{r_{1}, r_{1}+1\right\}$.

When $2 \leqslant r_{1}=r_{2}-1$, the ring $\mathbb{K}[G]$ is nearly Gorenstein and not Gorenstein.

Proof. By [10, Proposition 2.2], $\mathbb{K}[G]$ is isomorphic to the Hibi ring associated to the distributive lattice of order ideals in the poset $P$ which consists of two disjoint chains with $r_{1}-1$ and $r_{2}-1$ elements, respectively. By Theorem $4, \mathbb{K}[G]$ is nearly Gorenstein if and only if $r_{1}=1$, or $r_{1} \geqslant 2$ and $r_{2} \in\left\{r_{1}, r_{1}+1\right\}$.

For non-bipartite graphs we prove the following result.

Theorem 6. Let $R$ be the edge ring of a complete multipartite graph $K_{r_{1}, \ldots, r_{n}}$ with $n \geqslant 3$. The following statements are equivalent:

(i) $R$ is a Gorenstein ring;

(ii) $R$ is a nearly Gorenstein ring.

Proof. Clearly, $(i) \Rightarrow(i i)$. We'll prove the converse.

When $n=3$ and $r_{1}=1 \leqslant r_{2} \leqslant r_{3}$, by [10, Proposition 2.2] the ring $R$ is isomorphic to the Hibi ring associated to the distributive lattice of order ideals in a poset $Q$ with maximal chains $q_{1}<\cdots<q_{r_{1}}, q_{r_{1}+1}<\cdots<q_{r_{1}+r_{2}}$ and $q_{1}<q_{r_{1}+r_{2}}$. The poset $Q$ is connected, hence $R$ is nearly Gorenstein if and only if it is Gorenstein, i.e. $1=r_{1} \leqslant r_{2} \leqslant r_{3} \leqslant 2$.

We now consider the remaining cases: either $n=3$ and $r_{1} \geqslant 2$, or $n \geqslant 4$. Assume, by contradiction that $R$ is nearly Gorenstein and not Gorenstein, i.e.

$$
\operatorname{tr}\left(\omega_{R}\right)=\mathfrak{m}_{R}
$$

The monomials in $R$ and $\omega_{R}$ have a nice combinatorial description as feasable integer solutions to some systems of inequalities. This can be described as follows. We denote $H=\sum_{\{i, j\} \in E(G)} \mathbb{N}\left(\mathbf{e}_{i}+\mathbf{e}_{j}\right) \subset \mathbb{N}^{d}$ the affine semigroup generated by the columns of the vertex-edge incidence matrix for $G$, and $\mathcal{C}=\mathbb{R}_{+} H$ the rational cone over $H$.

For $\mathbf{u}=\left(u_{1}, \ldots, u_{d}\right) \in \mathbb{N}^{d}$, it follows from [13] and [18, Proposition 3.4] that $\mathbf{u} \in H$ (equivalently, $\mathbf{x}^{\mathbf{u}} \in R$ ) if and only if

$$
\begin{aligned}
\sum_{i=1}^{d} u_{i} & \equiv 0 \bmod 2, \\
u_{1}, \ldots, u_{d} & \geqslant 0, \quad \text { and } \\
\sum_{i \notin V_{k}} u_{i} \geqslant \sum_{j \in V_{k}} u_{j} \text { for all } k & =1, \ldots, n .
\end{aligned}
$$

The latter inequalities are equivalent to

$$
\sum_{i=1}^{d} u_{i} \geqslant 2 \sum_{j \in V_{k}} u_{j}, \text { for } k=1, \ldots, n .
$$


Since $R$ is normal, by [4], [17] (see also [2, Theorem 6.3.5(b)]), a $\mathbb{K}$-basis for $\omega_{R}$ is given by the monomials $\mathbf{x}^{\mathbf{u}}$ where $\mathbf{u}=\left(u_{1}, \ldots, u_{d}\right) \in \mathbb{Z}^{d}$ satisfies

$$
\begin{array}{r}
\sum_{i=1}^{d} u_{i} \equiv 0 \bmod 2, \\
u_{1}, \ldots, u_{d} \geqslant 1, \text { and } \\
\sum_{i=1}^{d} u_{i} \geqslant 2+2 \sum_{j \in V_{k}} u_{j}, \text { for } k=1, \ldots, n .
\end{array}
$$

From the equations above it is easy to see that if the monomial $\mathbf{x}^{\mathbf{u}}$ is in $R$ or in $\omega_{R}$, we can permute the exponents $x_{i}$ and $x_{j}$ whenever $i, j \in V_{k}$ for some $k$, and we obtain another monomial in $R$, or in $\omega_{R}$, respectively.

In what follows $\mathbf{u}=\left(u_{1}, \ldots, u_{d}\right)$ and $\mathbf{v}=\left(v_{1}, \ldots, v_{d}\right)$.

For a monomial $\mathbf{x}^{\mathbf{u}} \in \omega_{R}$ and $1 \leqslant k \leqslant n$ we say that $V_{k}$ (or simply, $k$ ) is a heavy component in $\mathbf{u}$ if

$$
\sum_{i=1}^{d} u_{i}=2+2 \sum_{j \in V_{k}} u_{j} .
$$

Claim 7. For any $\mathbf{x}^{\mathbf{u}} \in \omega_{R}$ there exist at most two heavy components in $\mathbf{u}$. In particular, there is at least one non-heavy component in $\mathbf{u}$.

Proof. Indeed, if $k_{1}<k_{2}<k_{3}$ are heavy components in $\mathbf{u}$, then by adding the equations (7) for these indices we get

$$
3 \sum_{i=1}^{d} u_{i}=6+\sum_{j \in V_{k_{1}} \cup V_{k_{2}} \cup V_{k_{3}}} 2 u_{j},
$$

If $n=3$, then $\sum_{i=1}^{d} u_{i}=6$. Since $u_{i} \geqslant r_{i} \geqslant 2$ for all $i$, we infer that $r_{1}=r_{2}=r_{3}=2$, and $\mathbb{K}[G]$ is a Gorenstein ring (by Theorem 1), which is not the case.

If $n \geqslant 4$, then $\sum_{i=1}^{d} u_{i}<6$. As $\sum_{i=1}^{d} u_{i}$ is even, we get that $n=4$ and $r_{1}=r_{2}=r_{3}=$ $r_{4}=1$. Example 2 implies that $R$ is a Gorenstein ring, which is false.

Claim 8. For any $1 \leqslant i \leqslant d$ there exists a monomial $\mathbf{x}^{\mathbf{u}} \in \omega_{R}$ such that $u_{i}=1$.

Proof. We fix $i$ and we denote $a_{i}=\min \left\{u_{i}: \prod x_{i}^{u_{i}} \in \omega_{R}\right\}$. By (5), $a_{i} \geqslant 1$. Assume $a_{i} \geqslant 2$, and say $i \in V_{k}$.

If $r_{k}>1$, we may pick $j \in V_{k}, j \neq i$. Then it is easy to check that the monomial $m=\frac{\mathbf{x}^{\mathbf{u}}}{x_{i}} x_{j} \in \omega_{R}$ and $\operatorname{deg}_{x_{i}}(m)=a_{i}-1$, a contradiction.

When $r_{k}=1$, then $n \geqslant 4$ and by the previous claim there is at least one non-heavy component $V_{k_{1}}$ in $\mathbf{u}$ which is different from $V_{k}$. We pick $j \in V_{k_{1}}$ and since the monomial $m=\frac{\mathbf{x}^{\mathbf{u}}}{x_{i}} x_{j} \in \omega_{R}$ and $\operatorname{deg}_{x_{i}}(m)=a_{i}-1$ we obtain a contradiction. 
It follows at once that

$$
\operatorname{gcd}\left(\mathbf{x}^{\mathbf{u}}: \mathbf{x}^{\mathbf{u}} \in \omega_{R}\right)=\prod_{i=1}^{d} x_{i}
$$

where the greatest common divisor is computed in the polynomial ring $S=\mathbb{K}\left[x_{1}, \ldots, x_{d}\right]$.

Since $\omega_{R}$ is generated by monomials, one gets that $\omega_{R}^{-1}$ is also generated by monomials in $\mathbb{K}\left[x_{1}^{ \pm 1}, \ldots, x_{d}^{ \pm 1}\right]$. If $f=\mathbf{x}^{\mathbf{u}} / \mathbf{x}^{\mathbf{v}} \in \omega_{R}^{-1}$ with $\mathbf{x}^{\mathbf{u}}$ and $\mathbf{x}^{\mathbf{v}}$ coprime monomials in $S$, then $\mathbf{x}^{\mathbf{v}}$ divides the greatest common divisor of the monomials in $\omega_{R}$. Hence, in order to determine a system of generators for the $R$-module $\omega_{R}^{-1}$ it is enough to scan among the (non-reduced) fractions $f=\mathbf{x}^{\mathbf{u}} /\left(x_{1} \ldots x_{d}\right)$, where $\mathbf{x}^{\mathbf{u}}$ is in the set

$$
\mathcal{B}=\left\{\mathbf{x}^{\mathbf{u}} \in S: \sum_{i=1}^{d} u_{i} \equiv 0 \bmod 2, \quad \mathbf{x}^{\mathbf{u}} \cdot \omega_{R} \subseteq x_{1} \ldots x_{d} R\right\} .
$$

A monomial $\mathbf{x}^{\mathbf{u}}$ is in $\mathcal{B}$ if and only if $\sum_{i=1}^{d} u_{i} \equiv 0 \bmod 2$ and

$$
x_{1}^{u_{1}+v_{1}-1} \cdots x_{d}^{u_{d}+v_{d}-1} \in R
$$

for all $x_{1}^{v_{1}} \cdots x_{d}^{v_{d}}$ in $\omega_{R}$. That is equivalent, via (2), (4), (3), to the fact that

$$
\begin{array}{r}
\sum_{i=1}^{d} u_{i} \equiv d \bmod 2, \text { and } \\
\sum_{i=1}^{d} u_{i}+\sum_{i=1}^{d} v_{i} \geqslant d-r_{k}+2 \sum_{j \in V_{k}} u_{j}+2 \sum_{j \in V_{k}} v_{j}, \\
\text { for } k=1, \ldots, d, \text { and any } \mathbf{x}^{\mathbf{v}} \in \omega_{R} .
\end{array}
$$

For $k=1, \ldots, n$ we set

$$
E_{k}=\min \left\{\sum_{i=1}^{d} v_{i}-2 \sum_{j \in V_{k}} v_{k}: \mathbf{x}^{\mathbf{v}} \in \omega_{R}\right\} .
$$

Therefore, (9) is equivalent to

$$
\sum_{i=1}^{d} u_{i} \geqslant d-r_{k}-E_{k}+2 \sum_{j \in V_{k}} u_{j} \text { for } k=1, \ldots, n .
$$

Before computing $E_{k}$ we make a simple observation regarding $d$ and the $r_{i}$ 's.

Claim 9. $2 r_{i}+2 \leqslant d$ for all $i=1, \ldots, n-1$.

Proof. Indeed, if that were not the case, then $2 r_{n}+2 \geqslant 2 r_{n-1}+2>d$, hence $2 r_{n} \geqslant$ $2 r_{n-1} \geqslant d-1$. This implies $r_{n}+r_{n-1} \geqslant d-1$, equivalently that $1=\sum_{i=1}^{n-2} r_{i}$, which is not possible in our setup. 
Next we show that $E_{k}$ does not depend on $k$.

Claim 10. $E_{k}=2$ for $k=1, \ldots, n$.

Proof. We fix $1 \leqslant k \leqslant n$. Clearly, $E_{k} \geqslant 2$, by (6). Then $E_{k}=2$ once we find

$$
\mathbf{x}^{\mathbf{v}} \in \omega_{R} \text { such that } \sum_{i=1}^{d} v_{i}=2+2 \sum_{j \in V_{k}} v_{j} .
$$

Using Eqs. (4), (5), (6), and translating $v_{i}=r_{i}+s_{i}$ for $i=1, \ldots, n$, we observe that finding $\mathbf{v}$ as in (11) is equivalent to finding integers $s_{1}, \ldots, s_{n}$ such that

$$
\begin{array}{r}
s_{1}, \ldots, s_{n} \geqslant 0 \\
\sum_{i=1}^{n} s_{i} \geqslant 2 s_{\ell}+2 r_{\ell}+2-d, \text { for } 1 \leqslant \ell \leqslant n, \ell \neq k, \text { and } \\
\sum_{i=1}^{n} s_{i}=2 s_{k}+2+2 r_{k}-d .
\end{array}
$$

The $s_{\ell}$ represents the sum of the components of $\mathbf{v}$ from $V_{\ell}$, each decreased by one. Note that (14) already implies that $\sum_{i=1}^{n} s_{i} \equiv d \bmod 2$.

We have two cases to consider.

Case $k=n$ :

We let $s_{\ell}=\lfloor d / 2\rfloor-r_{\ell}-1$ for $\ell=1, \ldots, n-1$. For $(14)$ to hold, we must let

$$
\begin{aligned}
s_{n} & =\sum_{i=1}^{n-1} s_{i}-2-2 r_{n}+d=(n-1)\lfloor d / 2\rfloor-d+r_{n}-(n-1)-2-2 r_{n}+d \\
& =(n-1)(\lfloor d / 2\rfloor-1)-r_{n}-1 \geqslant 2(\lfloor d / 2\rfloor-1)-r_{n}-1 \geqslant d-r_{n}-2 \geqslant 0 .
\end{aligned}
$$

For $\ell<n$, one has $s_{\ell} \geqslant 0$ by the previous Claim. Also, $2 s_{\ell}+2+2 r_{\ell}-d$ is either 0 or 1 , depending on $d$ being even or odd. Therefore, (13) and (12) are all verified.

Case $1 \leqslant k \leqslant n-1$ :

We let $s_{n}=0$ and $s_{\ell}=\lfloor d / 2\rfloor-r_{\ell}-1$ for $\ell=1, \ldots, n-1$ where $\ell \neq k$. For (14) to hold, we must let

$$
s_{k}=\left(\sum_{1 \leqslant i \leqslant n-1, i \neq k} s_{i}\right)+s_{n}-2-2 r_{k}+d
$$

Clearly, $s_{k} \geqslant 0$ since $d \geqslant 2 r_{k}+2$. Arguing as in the other case, for $k \neq \ell<n$ one has $s_{\ell} \geqslant 0$ and (13) holds. We are left to verify that

$$
\sum_{i=1}^{n} s_{i} \geqslant 2 s_{n}+2 r_{n}+2-d .
$$


Substituting (14) into the left hand side term above, (16) is equivalent to

$$
s_{k}+r_{k} \geqslant s_{n}+r_{n}
$$

Using (15) we get that

$$
\begin{aligned}
s_{k}+r_{k} & =\left(\sum_{1 \leqslant i \leqslant n-1, i \neq k} s_{i}\right)+s_{n}+d-r_{k}-2 \\
& =\left(\sum_{1 \leqslant i \leqslant n-1, i \neq k} s_{i}\right)+s_{n}+r_{n}+\left(d-r_{k}-r_{n}-2\right) \geqslant s_{n}+r_{n},
\end{aligned}
$$

where for the latter inequality we used the observation that $d \geqslant r_{k}+r_{n}+2$ in our setup. Consequently, $s_{1}, \ldots, s_{n}$ fulfil (12), (13), (14), and $E_{k}=2$.

We can now finish the proof of Theorem 6 .

Let $m=x_{1}^{a_{1}} \ldots x_{d}^{a_{d}}$ be a monomial generator for $\omega_{R}$. Then $\operatorname{deg} m=\sum_{i=1}^{d} a_{i} \geqslant$ $2+2 \sum_{j \in V_{k}} a_{j}$ for all $k=1, \ldots, n$. In particular, $\operatorname{deg} m \geqslant 2 r_{n}+2$.

Let $f=\mathbf{x}^{\mathbf{u}} /\left(x_{1} \cdots x_{d}\right)$ be a monomial in $\omega_{R}^{-1}$, with $\mathbf{x}^{\mathbf{u}} \in \mathcal{B}$. By $(10)$,

$$
\operatorname{deg} \mathbf{x}^{\mathbf{u}}=\sum_{i=1}^{d} u_{i} \geqslant d-r_{k}-2+2 \sum_{j \in V_{k}} u_{j} \text { for all } k=1, \ldots, n .
$$

Since $d>r_{n}+2$ in our setup, we find a component $k_{1}$ such that $\sum_{j \in V_{k_{1}}} u_{j}>0$.

The product $m \cdot f$ is a monomial in $R$ of degree at least

$$
\left(2 r_{n}+2\right)+\left(d-r_{k_{1}}-2+2 \sum_{j \in V_{k_{1}}} u_{j}\right)-d \geqslant 2 r_{n}-r_{k_{1}}+2 \geqslant 3 .
$$

Consequently, $\operatorname{tr}\left(\omega_{R}\right)=\omega_{R} \cdot \omega_{R}^{-1} \subsetneq \mathfrak{m}_{R}$, a contradiction with (1).

\section{$2 \quad$ Stable set rings}

In this section we consider an algebra generated by the monomials coming from the stable sets of a graph.

Let $G$ be a finite simple graph on $[n]$ and $E(G)$ is the set of edges of $G$. A subset $C \subset[n]$ is a clique of $G$ if $\{i, j\} \in E(G)$ for all $i, j \in C$ with $i \neq j$. A subset $W \subset[n]$ is stable in $G$ if $\{i, j\} \notin E(G)$ for all $i, j \in W$ with $i \neq j$. In particular, the empty set as well as each $\{i\} \subset[n]$ is both a clique of $G$ and a stable subset of $G$. Let $\Delta(G)$ denote the clique complex of $G$ which is the simplicial complex on $[n]$ consisting of all cliques of $G$. Let $\delta$ denote the maximal cardinality of cliques of $G$. Thus $\operatorname{dim} \Delta(G)=\delta-1$. We say that $G$ is pure if $\Delta(G)$ is a pure simplicial complex, i.e. the cardinality of each maximal clique of $G$ is $\delta$. The chromatic number of a graph is the smallest number of colors that can be used for its vertices such that no adjacent vertices have the same color. The graph 
$G$ is called perfect if for all induced subgraphs $H$ of $G$, including $G$ itself, the chromatic number is equal to the maximal cardinality of cliques contained in $H$, see [1, p. 165].

Let $\mathbb{K}\left[x_{1}, \ldots, x_{n}, t\right]$ denote the polynomial ring in $n+1$ variables over the field $\mathbb{K}$. If, in general, $W \subset[n]$, then $x^{W} t$ stands for the squarefree monomial

$$
x^{W} t=\left(\prod_{i \in W} x_{i}\right) \cdot t \in \mathbb{K}\left[x_{1}, \ldots, x_{n}, t\right]
$$

Let $\operatorname{Stab}_{\mathbb{K}}(G)$ denote the subalgebra of $\mathbb{K}\left[x_{1}, \ldots, x_{n}\right]$ which is generated by those $x^{W} t$ for which $W$ is a stable set of $G$. Letting $\operatorname{deg}\left(x^{W} t\right)=1$ for any stable set $W$, the algebra $\operatorname{Stab}_{\mathbb{K}}(G)$ becomes standard graded. We call $\operatorname{Stab}_{\mathbb{K}}(G)$ the stable set ring of $G$.

It is known [15, Example 1.3 (c)] that $\operatorname{Stab}_{\mathbb{K}}(G)$ is normal if $G$ is perfect. It follows that, when $G$ is perfect, $\operatorname{Stab}_{\mathbb{K}}(G)$ is spanned over $\mathbb{K}$ by those monomials $\left(\prod_{i=1}^{n} x_{i}^{a_{i}}\right) t^{q}$ with $\sum_{i \in C} a_{i} \leqslant q$ for each maximal clique $C$ of $G$. Furthermore, the canonical module $\omega_{\mathrm{Stab}_{\mathbb{K}}(G)}$ of $\operatorname{Stab}_{\mathbb{K}}(G)$ is spanned over $\mathbb{K}$ by those monomials $\left(\prod_{i=1}^{n} x_{i}^{a_{i}}\right) t^{q}$ with each $a_{i}>0$ and with $\sum_{i \in C} a_{i}<q$ for each maximal clique $C$ of $G$. Thus [16, Theorem 2.1 (b)] implies that $\operatorname{Stab}_{\mathbb{K}}(G)$ is Gorenstein if and only if $G$ is pure.

The following lemma captures a sufficient combinatorial condition for $\operatorname{Stab}_{\mathbb{K}}(G)$ to be nearly Gorenstein.

Lemma 11. Let $G$ be a finite simple perfect graph such that $\operatorname{Stab}_{\mathbb{K}}(G)$ is nearly Gorenstein. Then every connected component of $G$ is pure.

Proof. Assume $V(G)=[n]$. Denote $R=\operatorname{Stab}_{\mathbb{K}}(G)$. Since each $x_{i} t$ as well as $t$ belongs to $R$, the quotient field of $R$ is the rational function field $\mathbb{K}\left(x_{1}, \ldots, x_{n}, t\right)$ over $\mathbb{K}$.

Suppose $G_{1}$ is a connected component of $G$ which is not pure. Let $\delta$ and $\delta_{1}$ denote the maximal cardinality of cliques of $G$ and of $G_{1}$, respectively. Then there is an edge $\left\{i_{0}, j_{0}\right\} \in E\left(G_{1}\right)$ for which $i_{0}$ belongs to a clique $C$ of $G$ with $|C|=\delta_{1}$ and for which $j_{0}$ belongs to no clique $C$ of $G$ with $|C|=\delta_{1}$.

Let $z=\prod_{i=1}^{n} x_{i}^{a_{i}^{\prime}} t^{q^{\prime}} \in \omega_{R}^{-1}$. Set $v_{1}=x_{1} \cdots x_{n} t^{\delta+1}$. It is easy to check that $v_{1} \in \omega_{R}$ and that each monomial belonging to $\omega_{R}$ is divisible (in $\mathbb{K}\left[x_{1}, \ldots, x_{n}, t\right]$ ) by $v_{1}$. Hence $a_{i} \geqslant-1$ for all $i$. Clearly, $x_{j_{0}} v_{1} \in \omega_{R}$ and $1 \neq x_{j_{0}} v_{1} z \in R$, hence $q^{\prime} \geqslant-\delta$.

Since $G$ is not pure, $R$ is not a Gorenstein ring and thus

$$
\operatorname{tr}\left(\omega_{R}\right)=\omega_{R} \cdot \omega_{R}^{-1}=\mathfrak{m}_{R}
$$

Let $w^{\prime}=\prod_{i=1}^{n} x_{i}^{a_{i}^{\prime}} t^{q^{\prime}} \in \omega_{R}^{-1}$ and $w=\prod_{i=1}^{n} x_{i}^{a_{i}} t^{q} \in \omega_{R}$ with $w^{\prime} w=x_{i_{0}} t$. Since $q^{\prime} \geqslant-\delta$ and $q \geqslant \delta+1$, one has $q^{\prime}=-\delta$ and $q=\delta+1$.

Let $v=x_{1} x_{2} \cdots x_{n} t^{\delta+1} \cdot x_{i_{0}}^{\delta-\delta_{1}}$. One has $v \in \omega_{R}$ and $x_{j_{0}} v \in \omega_{R}$. We claim that $w^{\prime} \cdot x_{j_{0}} v \in \mathfrak{m}_{R}$ is divisible by $x_{i_{0}} x_{j_{0}} t$, but it is not divisible by $t^{2}$. This is clear when $\delta>\delta_{1}$. In case $\delta=\delta_{1}$, since $i_{0}$ belongs to a clique $C$ of $G$ with $|C|=\delta$, one has $a_{i_{0}}=1$. Thus $a_{i_{0}}^{\prime}=0$ and the claim is verified.

Thus $w^{\prime} \cdot x_{j_{0}} v$ must be of the form $x^{W} t$, where $W$ is a stable set of $G$, which contradicts $\left\{i_{0}, j_{0}\right\} \in E(G)$. Hence $\mathfrak{m}_{R} \subsetneq \operatorname{tr}\left(\omega_{R}\right)$, as desired. 
Recall that the $a$-invariant of any graded algebra $R$ with canonical module $\omega_{R}$ is defined as $a(R)=-\min \left\{i:\left(\omega_{R}\right)_{i} \neq 0\right\}$.

Corollary 12. If $G$ is a perfect graph then $a\left(\operatorname{Stab}_{\mathbb{K}}(G)\right)=-\operatorname{dim} \Delta(G)-2$.

Proof. Let $\delta$ be the maximal size of a clique in $G$. From the proof of the Lemma 11, $v=x_{1} \cdots x_{n} t^{\delta+1}$ is in $\left(\omega_{\operatorname{Stab}_{\mathbb{K}}(G)}\right)_{\delta+1}$ and $v$ divides every monomial in $\omega_{\mathrm{Stab}_{\mathbb{K}}(G)}$. This gives the conclusion.

Theorem 13. Let $G$ be a finite simple graph with $G_{1}, \ldots, G_{s}$ its connected components and suppose that $G$ is perfect. Let $\delta_{i}$ denote the maximal cardinality of cliques of $G_{i}$. Then $\operatorname{Stab}_{\mathbb{K}}(G)$ is nearly Gorenstein if and only if each $G_{i}$ is pure and $\left|\delta_{i}-\delta_{j}\right| \leqslant 1$ for $1 \leqslant i<j \leqslant s$.

Proof. Suppose that $\operatorname{Stab}_{\mathbb{K}}(G)$ is nearly Gorenstein. It follows from Lemma 11 that each $G_{i}$ is pure and each $\operatorname{Stab}_{\mathbb{K}}\left(G_{i}\right)$ is Gorenstein. Since $\operatorname{Stab}_{\mathbb{K}}(G)$ is the Segre product of $\operatorname{Stab}_{\mathbb{K}}\left(G_{1}\right), \ldots, \operatorname{Stab}_{\mathbb{K}}\left(G_{s}\right)$, it follows from [7, Corollary 4.16] and [8, Corollary 2.8] that

$$
\left|a\left(\operatorname{Stab}_{\mathbb{K}}\left(G_{i}\right)\right)-a\left(\operatorname{Stab}_{\mathbb{K}}\left(G_{j}\right)\right)\right| \leqslant 1 \text { for all } i, j .
$$

Corollary 12 yields $\left|\delta_{i}-\delta_{j}\right| \leqslant 1$ for $1 \leqslant i<j \leqslant s$. Furthermore, the "If" part also follows from [7, Corollary 4.16] and [8, Corollary 2.8].

Corollary 14. Let $G$ be a finite simple graph which is pefect and connected. Then the ring $\operatorname{Stab}_{\mathbb{K}}(G)$ is nearly Gorenstein if and only if it is Gorenstein.

\section{References}

[1] B. Bollobás, Modern Graph Theory, GTM 184, Springer, 1998.

[2] W. Bruns, J. Herzog, Cohen-Macaulay Rings, Revised Ed., Cambridge Stud. Adv. Math., vol. 39, Cambridge University Press, Cambridge, 1998.

[3] W. Bruns, W. Vasconcelos, R. Villarreal, Degree bounds in monomial subrings, Illinois Journal of Mathematics 41 (1997), no. 3, 341-353.

[4] V.I. Danilov, The geometry of toric varieties, Russian Math. Surveys, 33 (1978), $97-154$.

[5] E. De Negri, T. Hibi, Gorenstein Algebras of Veronese Type, J. Algebra 193 (1997), 629-639.

[6] S. Goto, R. Takahashi, N. Taniguchi, Almost Gorenstein rings-towards a theory of higher dimension, J. Pure Appl. Algebra 219 (2015), 2666-2712.

[7] J. Herzog, T. Hibi, D.I. Stamate, The trace of the canonical module, Israel J. Math. 233 (2019), 133-165.

[8] J. Herzog, F. Mohammadi, J. Page, Measuring the non-Gorenstein locus of Hibi rings and normal affine semigroup rings, J. Algebra 540 (2019), 78-99. 
[9] T. Hibi, Distributive lattices, affine semigroup rings and algebras with straightening laws, in: "Commutative Algebra and Combinatorics" (M. Nagata and H. Matsumura, Eds.), Advanced Studies in Pure Math., Volume 11, North-Holland, Amsterdam, 1987, pp. 93-109.

[10] A. Higashitani, K. Matsushita, Conic divisorial ideals and non-commutative crepant resolutions of edge rings of complete multipartite graphs, arXiv:2011.07714.

[11] A. Higashitani, K. Matsushita, Levelness versus almost gorensteinness of edge rings of complete multipartite graphs, arXiv:2102.02349.

[12] M. Hochster, Rings of invariants of tori, Cohen-Macaulay rings generated by monomials, and polytopes, Ann. of Math. 96 (1972), 318-337.

[13] H. Ohsugi, T. Hibi, Normal polytopes arising from finite graphs, J. Algebra 207 (1998), 409-426.

[14] H. Ohsugi, T. Hibi, Compressed polytopes, initial ideals and complete multipartite graphs, Illinois J. Math 44 (2000), 391-406.

[15] H. Ohsugi, T. Hibi, Convex polytopes all of whose reverse lexicographic initial ideals are squarefree, Proc. Amer. Math. Soc. 129 (2001), 2541-2546.

[16] H. Ohsugi, T. Hibi, Special simplices and Gorenstein toric rings, J. Combin. Theory, Ser. A 113 (2006), 718-725.

[17] R. Stanley, Hilbert functions of graded algebras, Adv. Math. 28 (1978), 57-83.

[18] R. H. Villarreal, On the equations of the edge cone of a graph and some applications, Manuscripta Math. 97 (1998), 309-317. 